\title{
Current management of transition of young people affected by rare renal conditions in the ERKNet
}

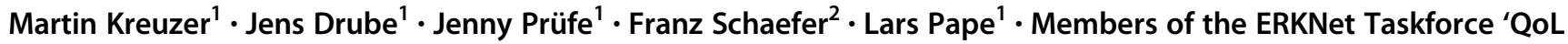 \\ \& Transition'
}

Received: 11 January 2019 / Revised: 12 April 2019 / Accepted: 11 June 2019 / Published online: 30 July 2019

(c) The Author(s), under exclusive licence to European Society of Human Genetics 2019

\begin{abstract}
Transition in medical care is a high-risk period in adolescence and young adulthood. To date, data on transition policy, its application in practice, and transition procedures in patients with rare, hereditary kidney diseases in Europe is scarce. An online survey was developed and was distributed within the paediatric centres of the European Reference Network for Rare Kidney Diseases (ERKNet) aiming to assess the transition-relevant structures from the providers' perspectives. Its items were based on the consensus statement on transition published by the International Society of Nephrology (ISN) and the International Paediatric Nephrology Association (IPNA) in 2011. Forty-six paediatric experts based at 28/32 ERKNet university hospitals participated. Annually, a median number of 14 patients (1-80) are transferred to adult based care. One centre continued to care for paediatric kidney transplant recipients throughout their entire lifespan. Choosing this option terminated the survey and no further data was obtained from this centre. 29/45 experts confirmed the application of an-at least unwritten - transition procedure (64\%). Transition clinics are offered by 23 experts. Most physicians (40\%) transfer patients at age 18-19, 10 experts at age <18. Most physicians transfer the patients to a university hospital and/or a community hospital. The transition guidelines have been implemented in ERKNet centres only partly and with huge heterogeneity. Implementation of transition tools and structures within ERKNet could improve health of children with hereditary kidney diseases. Adherence of experts to the transition-guidelines was significantly correlated with gross national income of their countries.
\end{abstract}

\section{Introduction}

A disease or disorder is defined as rare in Europe if it affects less than 1 in 2000 citizens. This comprises all paediatric chronic kidney diseases (CKD) and kidney transplantation (KTx). Conditions include among others: congenital malformations, familial cystic renal diseases, glomerulopathies, tubulopathies, microangiopathies, and metabolic

Supplementary information The online version of this article (https:// doi.org/10.1038/s41431-019-0460-z) contains supplementary material, which is available to authorized users.

Martin Kreuzer

kreuzer.martin@mh-hannover.de

1 Department of Paediatric Nephrology, Hepatology \& Metabolic Diseases, Hannover Medical School, Hannover, Germany

2 Department of Paediatrics I, University Children's Hospital, Heidelberg, Germany nephropathies all of which are mainly based on genetic abnormalities [1-3]. ERKNet is the "European Reference Network for Rare Kidney Diseases”, a consortium of 38 expert paediatric and adult nephrology centres in 12 European countries which provide healthcare to more than 40,000 patients with rare kidney conditions. Funded by the European Union, ERKNet is dedicated to improve knowledge about rare kidney diseases. It offers virtual consultation services to physicians, and actively supports clinical research. One goal of the ERKNet taskforce "QoL \& Transition" is to evaluate the transition structures in place in the ERKNet centres, and to develop and implement transition guidelines. As a first step, an online survey on current paediatric management of health care transition was conducted.

Health care transition is a purposeful process to prepare the paediatric patient to become a self-responsible adult patient who actively manages his/her condition in the adultcare setting. In adolescence and young adulthood brain development and maturation is a process which accounts for 
increased risk taking, impulsive behaviour, emotional instability, alienation, and non-adherence making this period equally challenging for patient and caregiver $[4,5]$. This turmoil interacts with the tasks put by CKD itself as well as its related co-morbidities which potentiates the struggle $[4,6]$. Developmental delay (especially in patients within CKD stages 4, 5, and those on dialysis) is common resulting in a substantial variability of the transitional requirements, and sometimes the need for longer-term transitional support into the adult care facility [6]. In addition, several demographic and socio-economic risk factors have been identified which may hamper successful transition, e.g. age, sex, language, income, family cohesion, and type of insurance [6-8]. This highlights the need for longterm transition programs with structured input from a multidisciplinary team of paediatric and adult nephrologists, youth workers, specialised nurses, psychologists and social workers $[6,9,10]$. Within the past decades, several nationwide or regional transition programs/procedures have been developed, e.g. "Ready Steady Go" (UK) [11], ON TRAC (British Columbia, Canada) [12], young adult clinic (Oxford, UK) [10], or the Zurich Transition Program (Zurich, Switzerland) [13].

Recent analyses show that transition programs involving a multi-disciplinary team can improve the outcomes post transition, but further research is needed [6, 14]. Most available evidence on the effectiveness of transition programs is limited by small sample sizes, short follow-up time, and the use of historical controls, thus limiting the validity of the conclusions which may be drawn [6]. While an international consensus on transition was published for paediatric kidney diseases in 2011, implementation in daily routines in Europe seems to be difficult $[9,15]$ and paediatric nephrology experts in Europe disagree on several transition topics [15]. We conducted a survey on current management of health care transition as a first step to understand the actual state of practice, and to prepare a consensus on recommendations within the ERKNet group which should subsequently be developed.

\section{Materials and methods}

The survey was developed by the ERKNet taskforce "QoL \& Transition" based on the 2011 ISN/IPNA consensus on transition. Hannover Medical School Ethics Committee waived the need for approval for the nature of the survey. The survey was approved by ERKNet.

An online-based cross-sectional survey was conducted. All paediatric ERKNet e-mail contacts were invited to complete the survey between July 28th and November 30th, 2017. Two reminders were sent 4 and 8 weeks after the first invitation. The survey is accessible as Supplementary
Online Data. The questionnaire started with items assessing centre characteristics such as size. Institution and city were asked to identify the participating centre. The survey was designed to assess data on three main questions: (1) Is there a transition process and how is it clinically implemented? (2) How is the transfer from paediatric to adultbased care managed and at what age? (3) What is the awareness of and adherence to the 2011 ISN/IPNA consensus statement on transition from paediatric to adult based health care?

A database including all the responses was provided by the questionnaire service for further analysis. A map showing countries and ERKNet centres is displayed at http://www.erknet.org.

To assess adherence of experts and centres with ISN/ IPNA-recommendations on transition the consensus paper was divided into 6 sections and a total of 18 items which were considered essential by the authors. Sections and items are available as Supplementary Online Data (Supplementary Table 1). We calculated an adherence-to-guidelines score based on the fulfilment of those 18 items. Confirming the use of each item in the survey scored one point. Some items were rated on a 5-point Likert scale and answers were graded from 0 (not at all) via $0.25,0.5,0.75$ to 1 (fully met). Maximum score was 18 points and adherence with consensus was considered very good (16-18 points), good (13-15 points), acceptable (10-12 points), and low ( $<9$ points).

We used data of the World Bank (http://www.worldba nk.org/) on gross national income from the year 2015 [16] for correlation and regression analysis for each country.

Since this survey was mainly designed to gather expert opinions, results are displayed per expert and not per centre -with two exceptions: (a) to describe centre characteristics and (b) for correlation of adherence scores with transition guidelines. In these cases, expert results were pooled for each centre.

Statistical analysis was performed using Prism 6.0 (GraphPad Software, Inc., La Jolla, USA). Continuous variables are expressed as median and range, as no normal distribution is assumed. Discrete variables are expressed as percentages. Chi-square test was used to compare groups. Spearman's coefficient was used for correlation analysis, as normal distribution was not assumed. Statistical significance was set at $p<0.05$.

\section{Results}

\section{Participating experts/centres}

A total of 46 experts of 28 centres participated in the survey: Belgium $(n=4)$, Czech Republic $(n=1)$, France 
$(n=5)$, Germany $(n=4)$, Italy $(n=14)$, Lithuania $(n=$ $2)$, The Netherlands $(n=5)$, Poland $(n=3)$, Spain $(n=$ $2)$, UK $(n=6)$. Experts from Sweden and Finland are unfortunately lacking (=6 experts). The ERKNet website listed 32 paediatric reference centres at the time the survey was performed. Therefore, $88 \%$ of paediatric reference centres participated with at least one expert. Most centres $(18 / 28)$ care for a large number $(>300)$ of children with rare chronic kidney disease (rCKD), 5/28 provide care for 100-300 children with rCKD. All centres are university hospitals. Daily clinics for children with rCKD are held at $21 / 28$ centres.

One expert at one centre declared that patients after paediatric KTx remained under paediatric care at all ages. Choosing this answer terminated the survey, so no further data was generated from this centre. One other centre (2 experts) stated that paediatric and adult nephrologists were working at the same facility (side by side)_-so technically there would be no real transfer. The remaining 16 centres transferred (median, range) 14 (1-80) patients per year from paediatric to adult based care.

\section{Transition policy and procedures}

$29 / 45$ experts $(64 \%)$ confirmed the existence of at least an unwritten transition agreement, plan or procedure at their centre. Seven out of 45 experts confirmed personal use of some kind of transition procedure, but not general use of the procedure at their centre; the further 9 experts denied having a transition procedure and subsequently skipped the survey part on transition. The remaining 36 experts continued to the transition section of the survey.

Table 1 Transition components and proportion of mention by ERKNet experts (multiple selections possible)
Components of transition and their respective frequency of mention are given in Table 1. Six experts ticked "other" and specified:

- "Specific program for patients after KTx."

- "At age 13-14, patients are seen by the transition urologist that has clinics in the Children's Hospital till age 18 and continues care in the adult hospital till age 30."

- Foyr experts listed "transition clinics" as "other".

One component of transition (as per ISN/INPA guidelines) is that "the concept of transition and goals in selfmanagement to acquire are explained [...] in early adolescence", marking the starting point of a transition program at age 12-14. Yet, about $70 \%$ (25/36) of experts commence their transition procedure much later than recommended (Fig. 1).



Fig. 1 Age of first introduction of the concept of transition of 36 ERKNet experts; the ISN/IPNA consensus recommendation is displayed as shaded area

\begin{tabular}{ll}
\hline Transition component & Proportion \\
\hline An unwritten plan for transition is made and communicated with the patient. & $23 / 36$ \\
A written transition plan is made and communicated with the patient. & $12 / 36$ \\
From a certain age the patient consults the nephrologist without a parent first-if applicable, & $19 / 36$ \\
the parent joins the consultation later on. & $6 / 36$ \\
Individual in-house training courses of transition relevant skills. & $12 / 36$ \\
Group training courses of transition relevant compentencies at your centre. & $6 / 36$ \\
External training of transition relevant skills (including inpatient rehab programs). & $11 / 36$ \\
Written information material about transition/transfer (e.g. handout, magazine, flyer, book...). & $2 / 36$ \\
Transition medical passport. & $2 / 36$ \\
Computer-based training/education (e.g. software, online/internet/intranet, app, etc.). \\
Progress of self-management skills is monitored by nephrologist/social worker WITHOUT & $11 / 36$ \\
special tools. & \\
Progress of self-management skills is monitored by a score-based system. & $5 / 36$ \\
Progress of self-management skills is monitored by questionnaire. & $6 / 36$ \\
Readiness of transfer is determined by using an appropriate tool (e.g. transition scale, & $5 / 36$ \\
questionnaire, self-rating). & \\
\hline
\end{tabular}


Table 2 ERKNet experts 5 level Likert scale rating median (range) on transition policy

\begin{tabular}{ll}
\hline Transition policy item & Rating (median, range) \\
\hline $\begin{array}{l}\text { In our centre information about transition is given to the patient in a gradual } \\
\text { manner appropriate to developmental state. }\end{array}$ & $5(3-5)$ \\
$\begin{array}{l}\text { The transition plan is individualised for each patient. } \\
\text { Young people are given the opportunity to participate in group sessions with }\end{array}$ & $3(2-5)$ \\
other young people who are about to transition, for peer support experience. & \\
\hline
\end{tabular}

Table 3 Proportion of groups involved into transition process by professionals besides the patient (multiple selections possible)

\begin{tabular}{ll}
\hline Group & Proportion \\
\hline Parents/Guardian & $36 / 36$ \\
Siblings & $6 / 36$ \\
Boyfriend/Girlfriend & $11 / 36$ \\
Peers/Friends & $2 / 36$ \\
Classmates/Co-workers & $0 / 36$ \\
Teacher/Instructor & $3 / 36$ \\
Other & $2 / 36$ \\
\hline
\end{tabular}

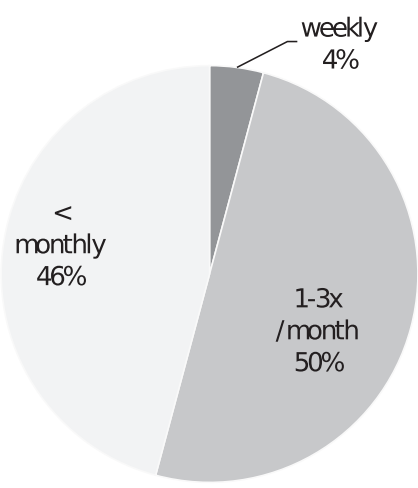

Fig. 2 Frequency transition clinics are held

The existence of at least one transition/transfer coordinator (nephrologist, social worker, nurse, etc.), who is responsible for management of all transition relevant issues, was confirmed by $21 / 36$ experts. Transition policy Likert scale medians are shown in Table 2. Frequency of other non-professionals involved in the transition process is shown in Table 3.

Transition clinics (e.g. alternating consultations with adult and paediatric nephrologist, shared consultations, a "young adult" clinic, etc.) were confirmed by $23 / 36$ experts. Frequency of transition clinics is shown in Fig. 2. All but one centre offer transition clinics specifically for their patients with CKD. In the majority of cases (16/23; multiple selections possible) transition clinics are organised as a shared consultation with adult and paediatric nephrologist side by side. Twelve experts hold alternating consultations with adult and paediatric nephrologist. Eight experts provide a "young adult clinic" with one physician who is specialised in the needs of adolescents and young adults. Three experts employ a specialist in transition medicine.

\section{Transfer age, policy, and procedure}

Different professions (e.g. paediatrician, psychologist, social-worker) actively involved in the process of transfer to adult-based care are available as Supplementary Online Data (Supplementary Table 2). 31/36 experts stated that the patient is offered the opportunity to informally visit the nominated adult service before transfer. Two experts chose "not applicable", because they work at the centre where paediatric and adult nephrologists are working at the same facility (side by side). Five-level Likert scale medians and ranges on transfer policy statements are shown in Table 4.

Patients are most commonly transferred to adult-based care at age 18-19 (18/45 experts); yet, 10/45 experts (22\%) frequently transfer patients younger than 18 years. A comparison of the actual age at transfer with the age considered most appropriate by the caring professionals is given in Fig. 3.

29/45 experts stated that transfer age is subject to regulation at their centre. In the majority of cases transfer-age was defined as age 18 years $(n=23)$; but also at age 15 $(n=1), 16(n=2), 19(n=2)$, and $20(n=1)$. Most commonly, $(n=14)$ age-regulations are placed by the care provider (e.g. hospital), followed by insurance company ( $n=11)$, physicians' association $(n=7)$, and the paediatric nephology unit itself $(n=4)$. One expert ticked "other" and specified: "paediatric urology department in conjunction with psychology". Multiple selections were possible for this question. 22/29 experts confirmed the possibility to postpone transfer beyond the defined age.

Finally, we asked to what kind of adult-based health care provider patients are transferred to (multiple selections possible). The most common answer was: university hospital $(n=43)$, followed by community hospital $(n=21)$, nephrologist's practice $(n=17)$, and general practitioner's office/ambulatory health care centres $(n=7)$.

\section{Adherence to ISN/IPNA consensus}

The 2011 ISN/IPNA consensus considered 18 components of transition and transfer as essential (see "Materials and methods" section). We scored the adherence to these 
Table 4 ERKNet experts 5 level Likert scale medians and ranges on transfer policy statements
Transfer policy item

Transfer at our centre is individualised for each patient after s/he has completed a transition plan; this will depend upon completion of physical growth and, where possible, educational, social and psychological attainment.

Transfer at our centre is agreed upon jointly by the patient and his/her family/ $4(1-5)$ carers in conjunction with the paediatric and adult renal care teams.

Transfer at our centre DOES NOT take place during a period of crises, especially $5(1-5)$ if there is unstable social support.

Transfer at our centre takes place after completing school education. $3(1-5)$

Transfer to adult renal care includes a comprehensive written summary (e.g. $\quad 5(1-5)$ referral letter) of all the multidisciplinary aspects of the young person's care.

We take into account treatment plans by other subspecialties (e.g. urological $5(1-5)$ supervision).
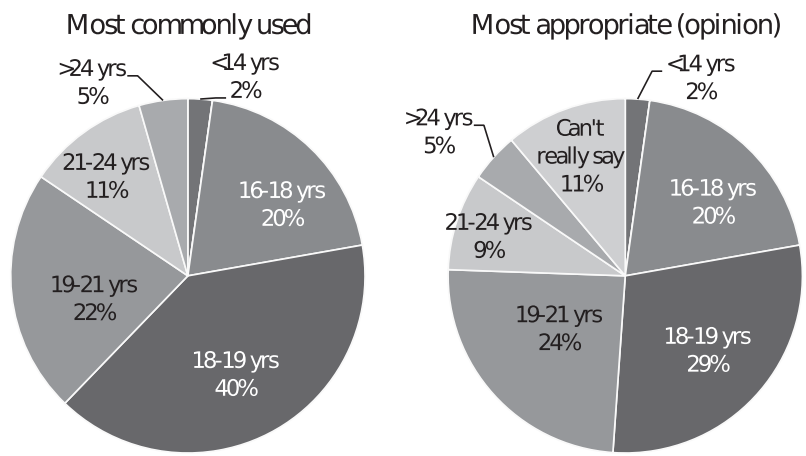

Fig. 3 Comparison of the actual transfer age with the age considered most appropriate by the caring ERKNet professionals

components of each participating centre with a maximum score of 18 points (one point per component). Median adherence score for all centres was 10.5 (range 3-17.75). A score below 9 was considered low adherence. We divided centres by location (East vs. West and South vs. North) and found no statistically significant North-South divide. However, Western centres median score of 10.5 was significantly higher than the scores of Eastern centres of 7.75 $(p<0.045)$. Centres with very good adherence (score 16 or higher) came from Belgium $(n=1)$, Germany $(n=1)$, and UK $(n=1)$.

The adherence score was significantly correlated with gross national income of the experts' countries (coefficient of correlation $0,63, p<0.001 ; r^{2}=0.29, p>0.005$; Fig. 4). There was no statistically significant correlation between centre size (number of patients under care) and score for adherence, nor did centre size and application of a transition procedure correlate.

\section{Discussion}

This survey sheds some light on current expert opinion about transition and transfer from paediatric to adult-based

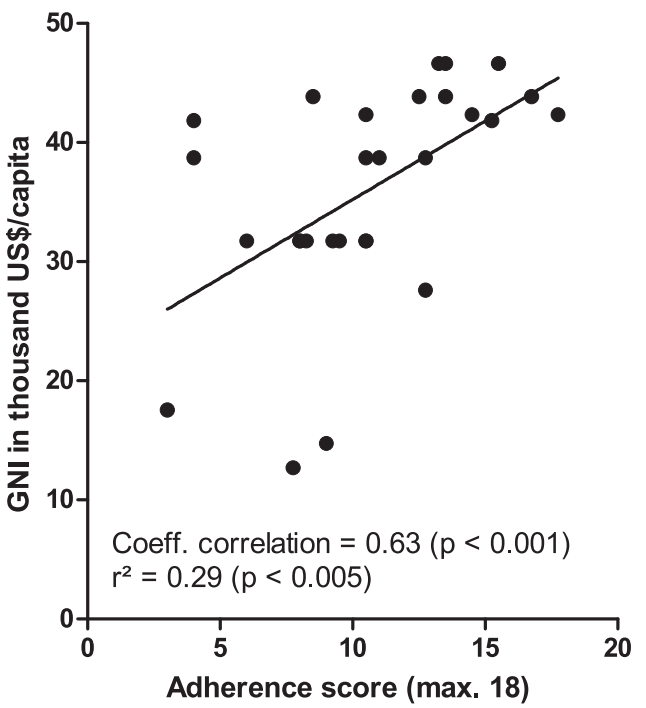

Fig. 4 Correlation of adherence to consensus score and gross national income

care in paediatric rCKD patients at ERKNet reference centres. It maps the current management of transition at these centres and indicates adherence with the international 2011 ISN/IPNA consensus guideline. We assume that the survey is representative because $88 \%$ of paediatric centres participated. The analysis of the survey identifies areas of transition that need work and improvement.

Transition is defined as a well-planned process that occurs over time leading the adolescent or young adult patient to gain competency. Its aim is to enable the patients so that at transfer they can take full responsibility for their own healthcare $[5,6,10]$. Therefore, the concept of transition and consequently the start of the learning process has to be introduced as early in adolescence as possible [11]. The patient is ready for transfer into adult based care if $s / h e$ knows and understands (a) his medical condition/illness, (b) his medication and other treatment, e.g. diet, (c) how/where to make appointments for medical care, and (d) how to react 
in emergency. Learning these competencies in the troubled period of puberty takes time. Implementation of a transition program like "Ready Steady Go" [11] in daily clinical routine is advisable in order to meet the milestones of transition over the developmental trajectory. A transition program like "Ready Steady Go" allows for monitoring the development of self-management skills in adolescent patients. In addition, it can be individualised for adolescents with impaired working memory and mental processing speed, developmental delay and reduced coping skills, which are frequently found in patients with higher CKD stages, on dialysis or after transplantation [6]. Several tools were developed and tested to monitor transfer readiness [17-21]. Using an appropriate tool in daily clinical practice also is advisable.

Only one-third of experts offered group training courses, e.g. therapeutic recreation camps, which have been proven to help knowledge transfer and to improve self-care skills $[13,22]$. Young people heavily rely on social media, internet and, computer/smartphone-based information [23]. A study from 2015 indicates that adolescents with chronic diseases predominantly prefer obtaining health information from the internet [5]. A prospective study in adult patients following lung transplantation showed a benefit on adherence with immunosuppressions using tablet-computer based education [24]. Thus, health care professionals caring for adolescents should keep up to date with information technology and social media.

Timing the transfer to adult based care is difficult and available data is scarce. One study showed that transfer below an age of 21 was associated with a higher risk for allograft loss in patients after KTx [25]. Age at transfer seems to be an important factor for successful transition. Two other studies reported that older age may be associated with improved readiness for transition in adolescents and young adults with CKD [7, 8]. Nonetheless, half of the experts stated that transfer-age at their centre is set to age 18 years. Transfer age may be regulated by insurance or physicians' associations. Both, actual transfer age and opinion on the most appropriate age for transfer vary widely in this survey. Further research in this area is needed. Although, a rigid regulation of transfer age seems to be counterproductive to an individualised successful transition. The frequent use of transition readiness scales, for example, could monitor whether patients are ready for adult care at time of transition.

Median score regarding adherence with the ISN/IPNA guidelines on health care transition in this survey is disappointing at low average. Interestingly, we found a significant correlation between adherence with the guidelines and gross national income of the experts' countries. Implementing guidelines into daily practice seems to depend at least partially on the availability of resources. On the other hand, these resources need to be directed to the improvement of transitional care. This can only be achieved if there are national efforts for directing money to transition and to properly reimburse transition programs. However, there may be other barriers to improving adherence to IPNA recommendations for transition. The authors of "Ready Steady Go" list seven common misconceptions preventing timely implementation of effective transition [11]. They elucidate that: (1) Definition of transition vs. transfer should be known. (2) One does not need a "transition clinic" to start the process. (3) Transition can start without the presence of an adult physician. (4) Tools for transition need not to be subspecialty specific. (5) Patients with learning disabilities can be involved in the transition process. (6) Transition should start at an age of about 11 years. (7) It is right to introduce the goal of an effective transition for patients with long-term or chronic conditions [11].

Our survey has some limitations: The results are based on an internet survey and not on personal interviews, making answers less reliable. Most ERKNet centres and countries are covered, yet the survey lacks input from Sweden and Finland, which can bias the results of the North-South divide statistics. However, practice of transition can vary widely even within the same country as recent studies show [15, 26-28].

We conclude that the 2011 ISN/IPNA guidelines on transition are implemented insufficiently in ERKNet paediatric nephrology centres. Therefore, development of a consensus within ERKNet centres is needed. In addition, there are further crucial issues to be addressed in the future including: (1) How is transition financed at the centres and how do European health care systems support transition of patients with special needs. (2) Information technology and social media should be implemented in patient training and education. The latter requires private data protection, which can be a problem with personal medical data. ERKNet can lead the way. Information for patients is already available at ERKNet homepage. It could be tailored to meet the needs of adolescents and young adults on their way through transition. Johnson et al. recently showed that youths with chronic conditions prefer obtaining health information from family/ parent and nurses/doctors-besides the internet [5]. It is possible to link these groups by use of information technology. ERKNet offers the potential of collaboration between paediatric and adult nephrologists. The participation of adult nephrologists in transition is crucial, but their views and prerequisites are under-explored. Finally, transfer should take place at an individual timing when transition readiness is achieved. Adapting a pre-existing transition readiness tool to the needs of rCKD patients and their caregivers could be helpful. Development of an ERKNet based transition readiness tool, transition guidelines, and a transition communication platform based on the results of this survey are planned to be implemented within ERKNet. 
Author contributions MK and LP conceived the survey and wrote the first draft of the manuscript. MK, FS, and LP designed the survey. MK performed the statistical analysis. MK, JD, JP, FS and LP participated in research design, read, revised, and approved the manuscript.

\section{Compliance with ethical standards}

Conflict of interest The authors declare that they have no conflict of interest.

Publisher's note: Springer Nature remains neutral with regard to jurisdictional claims in published maps and institutional affiliations.

\section{References}

1. Harambat J, van Stralen KJ, Kim JJ, Tizard EJ. Epidemiology of chronic kidney disease in children. Pediatr Nephrol. 2012; 27:363-73.

2. Leung JC. Inherited renal diseases. Curr Pediatr Rev. 2014;10:95-100.

3. Wenderfer SE, Gaut JP. Glomerular diseases in children. Adv Chronic Kidney Dis. 2017;24:364-71.

4. Foster BJ. Heightened graft failure risk during emerging adulthood and transition to adult care. Pediatr Nephrol. 2015;30:567-76.

5. Johnson MA, Javalkar K, van Tilburg M, Haberman C, Rak E, Ferris ME. The relationship of transition readiness, self-efficacy, and adherence to preferred health learning method by youths with chronic conditions. J Pediatr Nurs. 2015;30:e83-90.

6. Francis A, Johnson DW, Craig JC, Wong G. Moving on: transitioning young people with chronic kidney disease to adult care. Pediatr Nephrol. 2018;33:973-83.

7. Javalkar K, Fenton N, Cohen S, Ferris M. Socioecologic factors as predictors of readiness for self-management and transition, medication adherence, and health care utilization among adolescents and young adults with chronic kidney disease. Prev Chronic Dis. 2014;11:E117.

8. Javalkar K, Johnson M, Kshirsagar AV, Ocegueda S, Detwiler RK, Ferris M. Ecological factors predict transition readiness/selfmanagement in youth with chronic conditions. J Adolesc Health. 2016;58:40-6.

9. Forbes TA, Watson AR, Zurowska A, Shroff R, Bakkaloglu S, Vondrak K, et al. Adherence to transition guidelines in European paediatric nephrology units. Pediatr Nephrol. 2014;29:1617-24.

10. Harden PN, Walsh G, Bandler N, Bradley S, Lonsdale D, Taylor $\mathrm{J}$, et al. Bridging the gap: an integrated paediatric to adult clinical service for young adults with kidney failure. BMJ. 2012;344: e3718.

11. Nagra A, McGinnity PM, Davis N, Salmon AP. Implementing transition: ready steady go. Arch Dis Child Educ Pract Ed. 2015;100:313-20.

12. Paone MC, Wigle M, Saewyc E. TheON TRAC model for transitional care of adolescents. Prog Transplant. 2006;16:291-302.

13. Weitz M, Heeringa S, Neuhaus TJ, Fehr T, Laube GF. Standardized multilevel transition program: does it affect renal transplant outcome? Pediatr Transplant. 2015;19:691-7.
14. Coyne B, Hallowell SC, Thompson M. Measurable outcomes after transfer from pediatric to adult providers in youth with chronic illness. J Adolesc Health. 2017;60:3-16.

15. Kreuzer M, Prüfe J, Tönshoff B, Pape L. Survey on management of transition and transfer from pediatric- to adult-based care in pediatric kidney transplant recipients in Europe. Transplant Direct. 2018;4:e361.

16. The World Bank Group. GNI, Atlas Method (Current US\$). 2017. Available at http://data.worldbank.org/indicator/NY.GNI. ATLS.CD.

17. Anelli CG, Len CA, Terreri MTRA, Russo GCS, Reiff AO. Translation and validation of the Transition Readiness Assessment Questionnaire (TRAQ). J Pediatr. 2019;95:180-7.

18. Sawicki GS, Lukens-Bull K, Yin X, Demars N, Huang IC, Livingood $\mathrm{W}$, et al. Measuring the transition readiness of youth with special healthcare needs: validation of the TRAQ-Transition Readiness Assessment Questionnaire. J Pediatr Psychol. 2011;36:160-71.

19. Wood DL, Sawicki GS, Miller MD, Smotherman C, Lukens-Bull $\mathrm{K}$, Livingood WC, et al. The Transition Readiness Assessment Questionnaire (TRAQ): its factor structure, reliability, and validity. Acad Pediatr. 2014;14:415-22.

20. Zhang LF, Ho JS, Kennedy SE. A systematic review of the psychometric properties of transition readiness assessment tools in adolescents with chronic disease. BMC Pediatr. 2014;14:4.

21. Cohen SE, Hooper SR, Javalkar K, Haberman C, Fenton N, Lai H, et al. Self-management and transition readiness assessment: concurrent, predictive and discriminant validation of the STARx questionnaire. J Pediatr Nurs. 2015;30:668-76.

22. Watson A, Hilton D, Hackett D. Therapeutic recreation camps to provide a residential experience for young people in transition to adult renal units. Pediatr Nephrol. 2010;25:787-8.

23. Kreuzer M, Prufe J, Bethe D, Vogel C, Grosshennig A, Koch A, et al. The TRANSNephro-study examining a new transition model for post-kidney transplant adolescents and an analysis of the present health care: study protocol for a randomized controlled trial. Trials. 2014;15:505.

24. Suhling H, Rademacher J, Zinowsky I, Fuge J, Greer M, Warnecke $\mathrm{G}$, et al. Conventional vs. tablet computer-based patient education following lung transplantation-a randomized controlled trial. PLoS ONE. 2014;9:e90828.

25. Foster BJ, Platt RW, Dahhou M, Zhang X, Bell LE, Hanley JA. The impact of age at transfer from pediatric to adult-oriented care on renal allograft survival. Pediatr Transplant. 2011;15:750-9.

26. Prufe J, Dierks ML, Bethe D, Oldhafer M, Muther S, Thumfart J, et al. Transition structures and timing of transfer from paediatric to adult-based care after kidney transplantation in Germany: a qualitative study. BMJ Open. 2017;7:e015593.

27. Hattori M, Iwano M, Sako M, Honda M, Okada H, Akioka Y, et al. Transition of adolescent and young adult patients with childhood-onset chronic kidney disease from pediatric to adult renal services: a nationwide survey in Japan. Clin Exp Nephrol. 2016;20:918-25.

28. Webb N, Harden P, Lewis C, Tizzard S, Walsh G, Wray J, et al. Building consensus on transition of transplant patients from paediatric to adult healthcare. Arch Dis Child. 2010;95:606-11. 


\section{Affiliations}

Members of the ERKNet Taskforce 'QoL \& Transition'. Lars Pape ${ }^{1} \cdot$ Gema Ariceta ${ }^{3}$ - Larissa Kerecuk ${ }^{4}$. Giulia Bassanese $^{2} \cdot$ Franz Schaefer $^{2} \cdot$ Tanja Wlodkowski $^{2} \cdot$ Giovanni Capasso $^{5} \cdot$ Francesco Trepiccione $^{5}$. Natalie Biebuyck ${ }^{6} \cdot$ Pietro Manuel Ferraro $^{7}$. Flavia Galetti ${ }^{8} \cdot$ Antonia Bouts $^{8}$

1 Department of Paediatric Nephrology, Hepatology \& Metabolic Diseases, Hannover Medical School, Hannover, Germany

2 Department of Paediatrics I, University Children's Hospital, Heidelberg, Germany

3 Hospital Universitari Vall d'Hebron, Barcelona, Spain

4 Birmingham Women's and Children's Hospital, Birmingham, UK
5 UOC Nephrology and Dialysis - Università della Campania, Naples, Italy

6 Necker-Enfants Malades University Hospital, Paris, France

7 Gemelli Hospital, Rome, Italy

8 Department of Pediatric Nephrology, Emma Children's Hospital AMC, H 7-232, Meibergdreef 9, 1105 AZ Amsterdam, The Netherlands 